\title{
Evidence for the presence of dust in intervening QSO absorbers from the Sloan Digital Sky Survey
}

\author{
P. Khare ${ }^{1}$, D. G. York ${ }^{2}$, D. Vanden Berk ${ }^{3}$, V. P. Kulkarni ${ }^{4}$,
}

A. P. S. Crotts $^{5}$, D. E. Welty ${ }^{2}$, J. T. Lauroesch ${ }^{6}$, G. T. Richards ${ }^{7}$, Y. Alsayyad ${ }^{2}$, A. Kumar ${ }^{8}$, B. Lundgren ${ }^{9}$, N. Shanidze ${ }^{2}$, J. Vanlandingham ${ }^{2}$, B. Baugher ${ }^{10}$, P. B. Hall ${ }^{11}$, E. B. Jenkins ${ }^{7}$, B. Menard ${ }^{12}$, S. Rao ${ }^{13}$, D. Turnshek ${ }^{13}$ and C. W. Yip ${ }^{13}$

${ }^{1}$ Utkal University, India, ${ }^{2}$ University of Chicago, USA, ${ }^{3}$ Pennsylvania State University, USA, ${ }^{4}$ University of South Carolina, USA, ${ }^{5}$ University of Columbia, USA, ${ }^{6}$ Northwestern University, USA, ${ }^{7}$ Princeton University Observatory, USA, ${ }^{8}$ New York University, USA, ${ }^{9}$ University of Illinois, Urbana Champaign, USA, ${ }^{10}$ University of California, Santa Barbara, USA, ${ }^{11}$ York University, Canada, ${ }^{12}$ IAS, Princeton, USA, ${ }^{13}$ University of Pittsburgh, USA

Abstract. We find evidence for dust in the intervening QSO absorbers from the spectra of QSOs in the Sloan Digital Sky Survey Data Release 1. No evidence is found for the $2175 \AA$ feature which is present in the Milky Way dust extinction curve.

\section{Introduction}

Dust has long been suspected to be a constituent of QSO absorbers. The presence of dust in DLAs is suggested by the differential depletion of the refractory elements like $\mathrm{Cr}$ and Fe with respect to the volatile elements like Zn (Khare et al. 2004). [Cr/Zn] has been found to be correlated with E(B-V) in a sample of a few Sloan Digital Sky Survey (SDSS) QSOs (Khare et al. 2004). Yet another signature of the Milky Way type of dust, namely the $2175 \AA$ feature in the extinction curve has been detected in a few individual QSOs (e.g. Wang et al. 2004). Malhotra (1997) reported the detection of the $2175 \AA$ feature in the composite spectra of 96 QSOs. A much larger, homogeneous sample of QSOs is now available thanks to the SDSS (York et al. 2000). Here we study the SDSS Data Release 1 (DR1; Abazajian et al. 2003; Richards et al. 2002) having over 16,000 QSOs, for presence of dust in the intervening absorbers.

\section{Analysis and results}

(i) Sample definition: The absorption system identification has been done by the pipeline developed by York et al. (2005). To generate our "absorber" sample we collected all absorption systems with 4 or more detected lines outside the Lyman alpha forest, in non-BAL QSOs, and with (i) redshift between 1.0 and 1.9 (to ensure the observability of the $2175 \AA$ feature) (ii) $\mathrm{W}_{M g I I 2796}^{\text {rest }}>0.3 \AA$ and (iii) relative velocity w.r.t. the QSO $\beta>0.01$ (to ensure the intervening nature of the absorbers). The final sample consisted of 815 absorbers. We also constructed a "non-absorber" sample consisting of DR1 QSOs having no absorption systems in their spectra by matching $\mathrm{z}_{e m}$ and i-magnitude on a one to one basis between the "absorber" and "non-absorber" samples.

(ii) Composite spectra and evidence for reddening: The spectra of QSOs in the "absorber" sample were shifted to the absorber rest frame. The same shifts were applied 
to the spectra of the corresponding "non-absorber" QSOs. Geometric mean composite spectra for the two samples were generated. Fig. 1 shows the resulting spectra for the two samples and their ratio along with the best fit SMC extinction curve and the best fit Milky Way extinction curve. The presence of reddening in the "absorber" sample is clearly indicated. The $2175 \AA$ feature is definitely absent. We obtain a mean $\mathrm{E}(\mathrm{B}-\mathrm{V})=$ $0.013 \pm 0.002$ using the SMC extinction curve which seems to fit the spectra better.

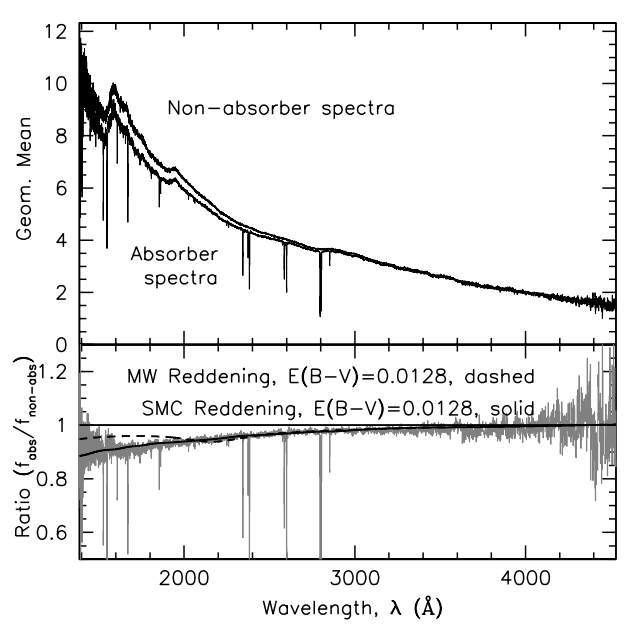

Figure 1. The upper panel shows the composite spectra in the absorber rest frame, of the "absorber" and the "non-absorber" samples. The lower panel shows the ratio of the two composite spectra and the best fit Milky Way and SMC extinction curves.

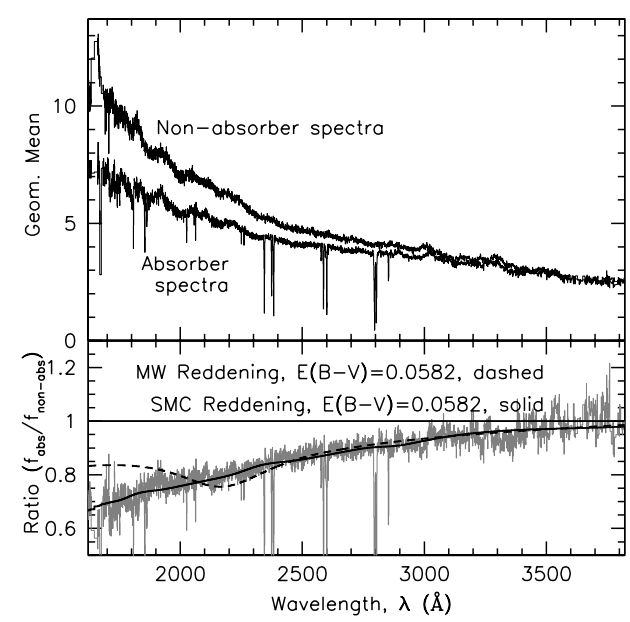

Figure 2. The upper panel shows the composite spectra in the absorber rest frame, of systems in which the Zn II/Cr II $\lambda 2062$ blend has been observed, for the "absorber" sample and the corresponding "non-absorber" sample. The lower panel shows the ratio of the two composite spectra and the best fit Milky Way and SMC extinction curves.

To study the dependence of reddening on the absorber properties, we have generated composite spectra for several "absorber" and "non-absorber" subsamples based on the strength of absorption lines of various species, on absorber redshifts, on $\beta$ and on the $\mathrm{i}$-magnitudes. The $\mathrm{E}(\mathrm{B}-\mathrm{V})$ values are found to be insensitive to $\beta$ and $\mathrm{i}$-magnitudes. We find that $\mathrm{E}(\mathrm{B}-\mathrm{V})$ is high for systems with strong $\mathrm{Mg}$ II $\lambda 2796$, while systems with weak $\mathrm{Mg}$ II $\lambda 2796$ show insignificant reddening. The $\mathrm{E}(\mathrm{B}-\mathrm{V})$ is highest $(0.058)$ for the sample of systems in which the Zn II/Cr II $\lambda 2062$ blend was detected (Fig. 2).

(iii) Colour excess: The colour excess of a QSO w.r.t. the mean colour $(\Delta(\mathrm{g}-\mathrm{i})$; the difference between the observed ( $g-i)$ for a given QSO and the median ( $g-i)$ for all QSOs at that redshift, Richards et al. 2003) is indicative of the extinction in its spectrum. Fig. 3 shows histograms for $\Delta$ (g-i) for the subsample of absorbers in which the Zn II/Cr II $\lambda 2062$ blend has been detected and for all the non-BAL QSOs in the Data Release 3 (DR3; Abazajian et al. 2005). The "absorber" subsample is significantly redder than average DR3 QSOs. Thus strong line absorbers appear to significantly redden QSOs. Fig. 4 shows the composite spectra and the fitted extinction curves for the subsample of QSOs with $\Delta(\mathrm{g}-\mathrm{i})>0.2$. A high value of $\mathrm{E}(\mathrm{B}-\mathrm{V})$ (0.081) was obtained for this sample. Note that the $\mathrm{E}(\mathrm{B}-\mathrm{V})$ values are likely to be overestimates due to the presence of multiple absorbers along some lines of sight and due to the fact that the absorber samples may not be complete due to the presence of low $\mathrm{S} / \mathrm{N}$ spectra in the sample. 


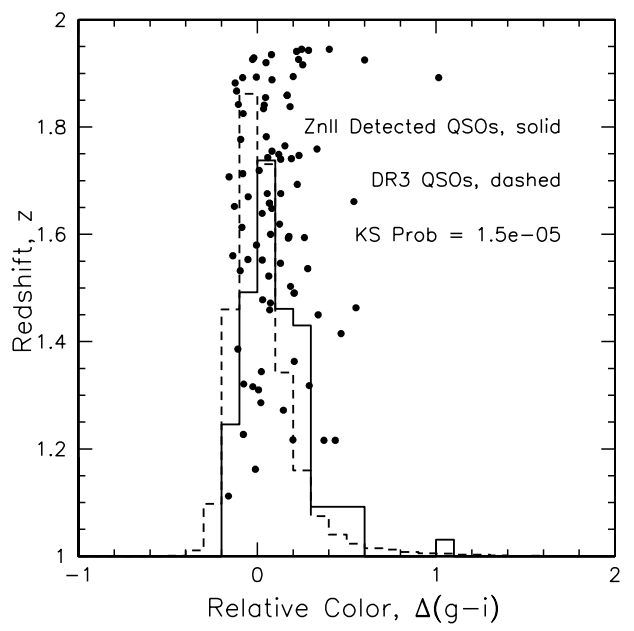

Figure 3. Histogram of the colour excess $\Delta$ (g-i) for the DR3 and the sample of systems in which the Zn II/Cr II $\lambda 2062$ blend was detected. Values of $\Delta(\mathrm{g}-\mathrm{i})$ for these systems have been plotted against their redshifts (dots). The KS probability that the two samples are drawn from the same populations is indicated.

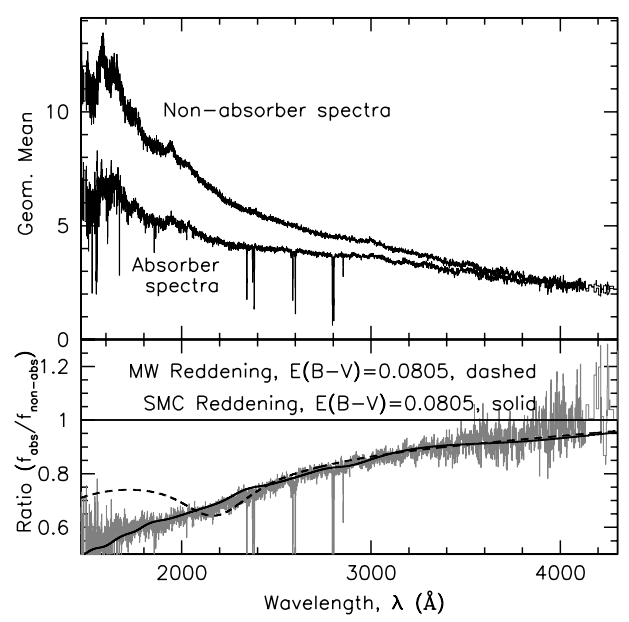

Figure 4. The upper panel shows the composite spectra in the absorber rest frame, of systems with $\Delta(\mathrm{g}-\mathrm{i})>0.2$, for the "absorber" sample and the corresponding "non-absorber" sample. The lower panel shows the ratio of the two composite spectra and the best fit Milky Way and SMC extinction curves.

\section{Conclusions}

- The intervening absorbers in the QSO spectra redden the spectra.

- The extinction curve due to the intervening absorbers is similar to the SMC extinction curve.

- No evidence is found for the presence of the $2175 \AA$ feature.

- The observed $\Delta$ (g-i) excess for QSOs with strong absorption systems appears to be a result of the reddening due to dust in the intervening absorbers.

\section{Acknowledgement}

Funding for the creation and distribution of the SDSS Archive has been provided by the Alfred P. Sloan Foundation, the Participating Institutions, the National Aeronautics and Space Administration, the National Science Foundation, the U.S. Department of Energy, the Japanese Monbukagakusho, and the Max Planck Society. The SDSS Web site is http://www.sdss.org/.

\section{References}

Abazajian, K., et al., 2003, AJ, 126, 2081

Abazajian, K., et al., 2005, AJ, 129, 1755

Khare, P., et al., 2004, ApJ, 616, 86

Malhotra, S., 1997, ApJ, 488, L101

Richards, G. T., et al., 2002, AJ, 123, 2945

Richards, G. T., et al., 2003, AJ, 126, 1131

Wang, J., Hall, P. B., Ge, J., Li, A., Schneider, D. P., 2004, ApJ, 609, 589

York, D. G., et al., 2000, AJ, 120, 1579

York, D. G., et al., 2005, this proceedings 